\title{
Super-latent inhibition with delayed conditioned taste aversion testing
}

\author{
L. G. DE LA CASA \\ University of Seville, Seville, Spain \\ and \\ R. E. LUBOW \\ Tel Aviv University, Tel Aviv, Israel
}

\begin{abstract}
In three conditioned taste aversion experiments with rats, latent inhibition (LI) was examined as a function of the time interval ( 1 or 21 days) between the conditioning and the test phases. In Experiments 1 and 2 , the effects of US intensity on LI were examined. LI increased in the 21-day condition, as compared with the 1-day condition, with medium and high US intensity, but not with weak US intensity. Groups not preexposed to the CS flavor had similar aversions when testing was conducted 1 day after conditioning, as compared with 21 days. In Experiment 3A, delay-induced super-LI was obtained when the delay was spent in the home cage and the experimental stages took place in a different context (as in Experiments 1 and 2). In Experiment 3B, when all the stages, including the delay period, were conducted in the home cage, there was no super-LI effect. The modulation of delay-induced super$\mathrm{LI}$ as a function of US intensity and context extinction is discussed in relation to association deficit and retrieval interference theories of $\mathrm{LI}$.
\end{abstract}

Passive exposures to a stimulus, whether it be a light, a tone, or a flavor, have a number of consequences. Of these, the most obvious is the gradual reduction in the magnitude of the unconditioned responses that are elicited by the repeated stimulus (habituation). However, more subtle effects can be seen when that same stimulus is subsequently paired with an unconditioned stimulus (US). Such pairings of a conditioned stimulus (CS) with a US are less effective in promoting conditioned performance than are those that are not preceded by passive exposure to the CS. This phenomenon, latent inhibition (LI), has been the subject of extensive research (see Lubow, 1989, for a review). The deficit in the performance of a learning task that results from such stimulus preexposure is found in many species and test paradigms.

LI is most often attributed to some mechanism operative during the preexposure period that affects the subsequent associability of the stimulus when it is paired with the US (Lubow, Weiner, \& Schnur, 1981; Mackintosh, 1975; Pearce \& Hall, 1980; Wagner, 1981). In opposition to the idea that LI represents an acquisition failure, it has been suggested that $\mathrm{LI}$ is a result of retrieval failure (e.g., Bouton, 1993; Miller, Kasprow, \& Schachtman, 1986). Such a hypothesis proposes that, following stimulus pre-

This research was supported by DGES Grant PB95-0529-C02-01 to the first author. Correspondence concerning this article should be addressed either to L. G. de la Casa, Department of Experimental Psychology, Avda. San Francisco Javier, s/n, 41005 Seville, Spain (e-mail: delacasa@cica.es), or to R. E. Lubow, Department of Psychology, Tel Aviv University, Ramat-Aviv 69978, Israel (e-mail: lubow@freud.tav. ac.il). exposure, the acquisition of the new association to the old stimulus proceeds normally. However, in the test stage, when the animal again encounters the target stimulus, two competing associations are retrieved: the earlier stimulusno-consequence association from the preexposure stage and the stimulus-US association from the acquisition stage. In normal LI, the nonpreexposed group exhibits greater conditioned response strength than does the preexposed group because there is only the second association to be retrieved, whereas the preexposed group exhibits less conditioned response strength, either because the first association is the one that is retrieved or because the first association interferes with the retrieval of the second one.

The primary evidence for the retrieval failure hypothesis of LI comes from studies that have varied the time between acquisition and test. If $L I$ is found after a short delay between acquisition and test stages, but not after a long delay, and if this difference is due to the preexposeddelay group's providing more evidence of conditioning than does the preexposed-no-delay group, this is presumptive evidence that with the short delay, the CS-US association was present but not manifest and that something occurred during the longer delay that allowed the originally encoded CS-US association to be retrieved. However, such an effect of delay has not been obtained consistently. With conditioned taste aversion (CTA), a reinstatement of conditioning (i.e., a loss of LI) with long acquisition-test intervals has been reported in several studies (Aguado, Symonds, \& Hall, 1994, Experiment 1; Bakner, Strohen, Nordeen, \& Riccio, 1991, Experiments 1 and 2; De La Casa \& Lubow, 1995). ${ }^{\prime}$ Other studies have obtained such an effect only when the test was conducted 
with a flavor that was different from the one in the preexposure and acquisition phases (Kraemer \& Ossenkopp, 1986; Kraemer \& Roberts, 1984; Kraemer \& Spear, 1992). Álvarez and López (1995, Experiment 2) also failed to find a diminution in LI after a long delay interval when the same flavor was used in preexposure, acquisition, and test.

Additional evidence for the retrieval failure hypothesis comes from CTA experiments that have imposed long delays between preexposure and conditioning stages, resulting in attenuated LI (Ackil, Carman, Bakner, \& Riccio, 1992; Aguado et al., 1994; Kraemer \& Roberts, 1984). However, as with the experiments manipulating the conditioning-test interval, the results are not completely consistent (Rosas \& Bouton, 1997).

The importance of LI in contemporary learning theory (Hall,1991; Mackintosh, 1983) compels one to confront the discrepant data and to uncover mechanisms/rules that would clarify the relationship between the two competing explanations of LI. In order to differentiate between acquisition failure and retrieval failure theories, one would need to (1) specify the variables that modulate LI, (2) identify the association(s) that are learned during the preexposure phase, and (3) postulate a mechanism by which these associations modulate test performance.

Both acquisition failure and retrieval failure theories of LI are based on the premise that something is learned during the preexposure period. They differ in terms of the locus of action of such learning. In the former case, what is learned in preexposure interferes with the development of a new association in the acquisition phase. In the latter case, such acquisition is unaffected by stimulus preexposure. Instead, the association that was learned in the preexposure phase and the association that was learned in the acquisition phase compete for expression in the test phase.

The effectiveness of these analyses is, however, dependent on specifying the associations that are learned in the preexposure and acquisition phases. For the acquisition phase, there is agreement that the organism acquires a CS-US association, and perhaps other associations as well, particularly to context. However, no such consensus exists for the identification of the associations acquired in the preexposure phase. Whether the association is CS-no-consequence, CS-context, context-noconsequence, or a higher order conditional association whereby the context becomes an occasion-setter for the expression of the CS-no-consequence association is, as yet, unresolved. Nor is it evident which, if any, of these possibilities is congruent with an acquisition failure or retrieval failure interpretation of LI.

Further theorizing on this issue would benefit from an analysis of the discrepant empirical data. To this end, we conducted three CTA experiments to clarify the role of particular variables on the LI attenuation that is mediated by the interpolation of a long delay between conditioning and test stages. In Experiments 1 and 2, the effects of US intensity on LI were examined as a function of conditioning-test delay. Unexpectedly, both experiments produced a delay-induced super-LI effect. In Experiment 3 , the role of the context in which the animal spends the delay interval was examined-namely, whether it is similar to or different from the context of preexposure, acquisition, and test. The results suggest that context extinction plays a role in producing super-LI.

\section{EXPERIMENT 1}

As was noted above, there are a number of different findings regarding the effects of long delays between acquisition and test stages on LI, almost all from CTA studies. These include LI attenuation (Aguado et al., 1994; Bakner et al., 1991; De la Casa \& Lubow, 1995) and LI attenuation only when the flavors in preexposure and conditioning are different from each other-LI remaining intact when the flavors are the same in all the stages (Kraemer \& Ossenkopp, 1986; Kraemer \& Roberts, 1984; Kraemer \& Spear, 1992; see also Álvarez \& López, 1995, Experiment 2).

Our first objective, then, was simply to replicate the LI attenuation effect, using the same flavor across all the experimental stages. Specifically, we sought to determine whether $\mathrm{LI}$ is reduced when a long delay interval (21 days) is imposed between the conditioning and the test stages. In addition, for reasons described below, we manipulated US intensity.

One possible explanation of the differences between experiments testing the effects of delay on LI is based on the retention interval effect (e.g., Batsell \& Best, 1992; Batsell \& George, 1996). This effect is exhibited as a greater taste aversion when the test is conducted 3 or more days after conditioning than when it is conducted 1 day after conditioning. Such an effect, if it disproportionately favors the flavor-preexposed group, as opposed to the nonpreexposed group, should be exhibited as a decrease in LI. Since the retention interval effect appears at medium and high US intensity levels, but not at low US intensity levels (Batsell \& George, 1996), this could explain some of the differences in results among the LI delay experiments. Indeed, Aguado et al. (1994) and Bakner et al. (1991), using intense USs (1\% of body weight, 0.3$\mathrm{M} \mathrm{LiCl}$, and $0.2 \%$ of body weight, $1-\mathrm{M} \mathrm{LiCl}$, respectively), found a disruption of LI with a 21-day delay, whereas Álvarez and López (1995, Experiment 2) did not find such an effect of delay with a weaker US ( $1 \%$ of body weight, $0.15-\mathrm{M} \mathrm{LiCl}$ ).

Experiment 1 tested the hypothesis that LI effects produced by acquisition-test delay are modulated by US intensity. Specifically, the increase of CTA with increased acquisition-test intervals, as reported by Batsell and George (1996) and others, suggests that LI should be reduced with the longer intervals and that this effect should be a positive function of US intensity.

To assess this prediction, a $2 \times 2 \times 2$ factorial design was employed. The first factor was preexposure (saccharin solution vs. water). The second factor was retention 
interval between the conditioning and the test phases (1 vs. 21 days). The third factor was low versus high US intensity $(0.5 \%$ body weight, $0.2-\mathrm{M}$ vs. $0.4-\mathrm{M}$ intraperitoneal [i.p.] $\mathrm{LiCl}$ ).

\section{Method}

Subjects. The subjects were 40 naive adult male Wistar rats, obtained from IFFA/CREDO Animals for Laboratory Service. The animals were individually housed in Plexiglas cages on a 12:12-h light:dark cycle. All testing occurred during the 12-h light period. Standard rat food was continuously available. Animal weights ranged from 290 to $365 \mathrm{~g}$.

Apparatus. Experimental sessions were conducted in an isolated room, different from the colony room. The subjects were housed in the colony room during the delay interval. For all the experimental sessions, the animals were transported to the experimental room, and each one was placed into a $30 \times 18 \times 18 \mathrm{~cm}$ Plexiglas cage, where preexposure, conditioning, and test trials were conducted. All the liquid rations were provided at room temperature in 150-ml graduated plastic bottles, fitted with stainless steel spouts, similar to those used in the home cages. The bottles were attached to the front of each cage during liquid presentations. The amount of liquid intake was indexed by the difference between bottle weight before and after the liquid presentation phase. The preexposure, conditioning, and test flavor was a $0.04 \%$ sodium saccharin solution. The US was a $0.5 \%$ of body weight i.p. LiCl injection, either 0.2 (low-intensity US) or $0.4 \mathrm{M}$ (high-intensity US).

Procedure. After 7 days on a 23.5-h water deprivation schedule, the animals were matched for body weight and assigned to eight groups. The stimulus preexposure stage was conducted on Days 8-11. All the sessions lasted $5 \mathrm{~min}$, and all liquid consumption was recorded. Half of the subjects were preexposed to saccharin (PE) and half to water (NPE). Conditioning was conducted on Day 12. All the animals received access to the saccharin solution for $5 \mathrm{~min}$, followed by injections of $\mathrm{LiCl}$. Half of the PE and NPE subjects were given an i.p. injection of the low-intensity US, and half of the highintensity US. The test was conducted on Days 13-17 for those groups in the 1-day delay condition and on Days 33-37 for those groups in the 21 -day delay condition. On each of the 5 test days, the subjects had a 5-min access to the saccharin solution. On Days 13-20, animals in the 21-day delay groups were given water ad lib. On Day 21 , the water deprivation schedule was reinstated to ensure that the subjects in both delay conditions were at the same deprivation level on the conditioning day.

\section{Results and Discussion}

On the conditioning day, the mean amount of saccharin consumed by the eight groups ranged from 6.82 to $9.90 \mathrm{ml}$. There were no significant differences between groups $[F(7,32)=1.66, p>.15]$. Subsequent analyses used the amount of saccharin consumed during the test stage as the index of conditioning.

A $5 \times 2 \times 2 \times 2$ analysis of covariance (ANCOVA) was performed on amount of saccharin solution ingested in the test stage, with main variables of trials (within subjects), preexposure (PE vs. NPE), delay ( 1 vs. 21 days), and US intensity (low vs. high). Amount of saccharin solution consumed on the conditioning day was used as a covariate. There was no main effect of, or interaction with, US intensity ( $p s>.32$, for all analyses that included the US variable), indicating that $\mathrm{LiCl}$ dosage did not affect CTA. Consequently, in subsequent analyses, the data from the two US intensity groups were collapsed.

Figure 1 (panel A) presents the mean amount of saccharin solution consumed over the five extinction trials as a function of preexposure and delay. A $5 \times 2 \times 2$ ANCOVA, with trials, preexposure, and delay as main

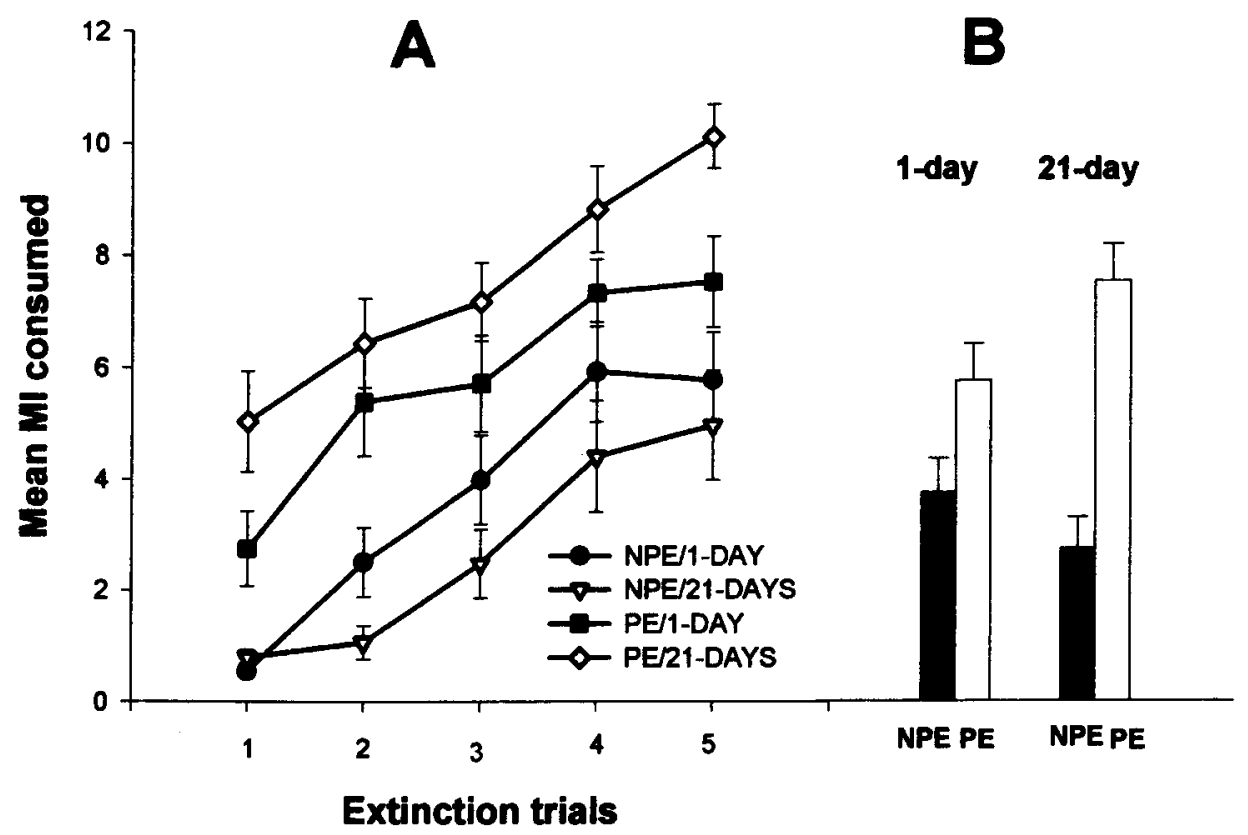

Figure 1. Mean amount of saccharin solution consumed by flavor-preexposed (PE) and flavornonpreexposed (NPE) groups as a function of delay between the conditioning and the test stages (1 and 21 days), on each of the five extinction day trials (panel $A$ ) and collapsed across trials (panel B). The unconditioned stimulus intensity variable was collapsed across conditions. 
variables, was conducted on these data. Again, amount of solution consumed during the conditioning day was a covariate. There was a main effect of trials $[F(4,140)=$ $79.35, p<.001]$. None of the interactions with trials was significant $(p s>.08)$. The between-subjects analysis revealed the usual LI effect, with the PE group drinking significantly more than the NPE group $[F(1,34)=51.67$, $p<.001]$. The main effect of delay was nonsignificant $(F<1)$. However, the preexposure $\times$ delay interaction was significant $[F(1,34)=9.77, p<.01]$.

The interaction is described in Figure 1 (panel B), where the mean amount of liquid consumption is averaged over all extinction days and displayed as a function of preexposure and delay conditions. As can be seen, the significant preexposure $\times$ delay interaction reflects more $\mathrm{LI}$ in the PE/21-day-delay group than in the PE/1-day-delay group. The expected retention interval effect was not obtained-that is, conditioned aversion in the long-delay NPE group was not greater than that in the short-delay NPE group.

\section{EXPERIMENT 2}

The results of Experiment 1 were completely unexpected. Instead of finding an attenuation of LI with a 21 day delay and an increase in that attenuation with a high US intensity, there was a super-LI effect; a 21-day delay, interpolated between the conditioning and the test phases, resulted in a larger LI effect than did a 1-day delay. In addition, LI was not modulated by US intensity.

Given these unanticipated results, we decided to replicate the basic design of Experiment 1. However, because the difference between $\mathrm{LiCl}$ dosages was relatively small in Experiment 1, Experiment 2 increased the difference in US intensities $(0.1$ and $0.5 \mathrm{M}$, instead of 0.2 and $0.4 \mathrm{M})$. Although our original hypothesis called for a reduction of LI in the PE/high-US/21-day group, as compared with the PE/low-US/21-day group, on the basis of the results of Experiment 1, we now anticipated a super-LI effect in the long-delay group, perhaps modulated by US intensity.

\section{Method}

Subjects. The subjects were 80 naive adult male Wistar rats, obtained from IFFA/CREDO Animals for Laboratory Service. Their weights ranged from 259 to $490 \mathrm{~g}$. The animals were individually housed in Plexiglas cages on a 12:12-h light:dark cycle. All testing occurred during the 12-h light period. Standard rat food was continuously available.

Apparatus and Procedure. The experiment was conducted in two replications, with 40 animals in each one. The apparatus and procedure were the same as those described for Experiment 1, except for the changes in US intensity. Specifically, the low-intensity US group received a $0.5 \%$ of bodyweight, $0.1-\mathrm{M} \mathrm{LiCl}$ i.p. injection, and the high-intensity US group a $0.5-\mathrm{M} \mathrm{LiCl}$ injection.

\section{Results and Discussion}

The mean amount of saccharin consumed by the eight groups on the conditioning day ranged from 8.38 to $9.88 \mathrm{ml}$. An analysis of variance, with groups and repli- cation as main factors, revealed a significant effect of replication $[F(1,64)=15.5, p<.001]$. The main effect of group and the interaction were not significant $(F \mathbf{s}<1)$. The effect of replication was due to the fact that the animals from the second replication drank more saccharin than did the animals from the first replication, probably as a result of greater body weight $-403 \mathrm{~g}(S D=42.34)$ and $340 \mathrm{~g}(S D=39.28)$, respectively.

A $5 \times 2 \times 2 \times 2 \times 2$ ANCOVA was performed on the amount of saccharin solution consumed during the test stage, with main variables of trials, preexposure, delay, US intensity, and replication. Amount consumed on the conditioning day was a covariate. Since there was no main effect of, or interaction with, replication ( $p s$ > .09 ), the data from both replications were combined in subsequent analyses.

Figure 2 shows mean saccharin consumption as a function of preexposure and delay for the low-US condition (panels A and B) and the high-US condition (panels C and D). Specifically, panels $A$ and $C$ show the mean amount of saccharin solution consumed across the five extinction trials, whereas panels $B$ and $D$ show mean saccharin consumption averaged over Test Days 1-5. A $5 \times 2 \times 2 \times 2$ ANCOVA, with amount consumed on conditioning day as covariate and main variables of trials, preexposure (PE vs. NPE), delay ( 1 vs. 21 days), and US intensity (low vs. high), was conducted. The main effect of trials was significant $[F(4,288)=180.75, p<.001]$. The trials $\times$ US-intensity and trials $\times$ preexposure interactions were significant $[F \mathrm{~s}(4,288)>7.40, p \mathrm{~s}<.001]$, reflecting the expected different rates of extinction as a function of US intensity and preexposure. The three-way interactions, trials $x$ preexposure $x$ delay and trials $X$ preexposure $\times$ US intensity, were also significant $\left[F_{\mathrm{s}}(4,288)>3.51, p \mathrm{~s}<.01\right]$, owing to faster extinction of the preexposure effect (LI) for the low-intensity condition than for the high-intensity condition and to faster extinction of the preexposure effect for the 21-day-delay condition than for the 1-day-delay condition. No other interaction was significant $(p s>.31)$. The between-subjects analysis revealed a significant main effect of preexposure $[F(1,71)=89.04, p<.001]$, indicating that preexposure to the saccharin solution produced weaker conditioning than did no preexposure. The main effect of delay was also significant $[F(1,71)=8.31, p<.01]$, owing to more liquid being consumed by the 21 -day-delay group than by the 1-day-delay group. Finally, the main effect of US intensity was significant $[F(1,71)=40.42, p<$ $.001]$, reflecting greater CTA with higher $\mathrm{LiCl}$ dosage. Importantly, the three-way interaction, preexposure $\times$ delay $\times$ US intensity, was significant $[F(1,71)=5.73$, $p<.05]$. No other interaction was significant ( $p s>.10$ ).

As can be seen in Figure 2 (panel B for the low-intensity condition, panel $\mathrm{D}$ for the high-intensity condition), the high-intensity condition promoted the expression of the delay factor, so that for the high- as compared with the low-intensity condition, there was a super-LI effect. This 

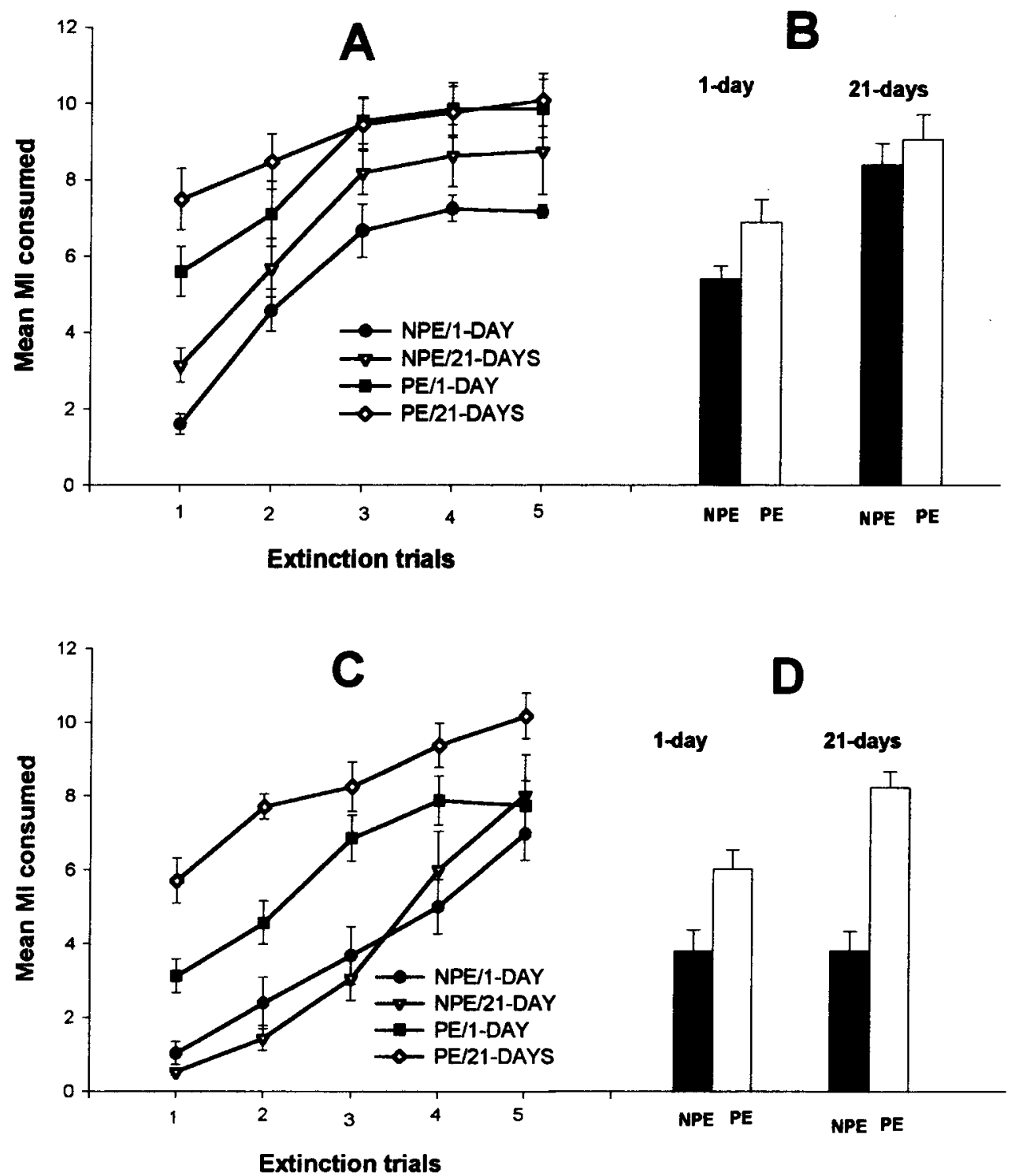

Figure 2. Mean amount of saccharin solution consumed by flavor-preexposed (PE) and flavornonpreexposed (NPE) groups as a function of delay between the conditioning and the test stages (1 and 21 days) for groups with a low-intensity unconditioned stimulus (US; upper panels) and a high-intensity US (lower panels). The left-hand panels $(A$ and $C$ ) present the data across the five extinction trials. The right-hand panels $(B$ and $D)$ present the data collapsed across trials.

impression was confirmed by two ANCOVAs, with preexposure and delay as main factors, one each for the low and the high US intensity groups. These analyses revealed a significant preexposure $\times$ delay interaction for the high-intensity condition $[F(1,35)=6.66, p<.05]$, but not for the low-intensity condition $[F(1,350)<1]$. Again, as in Experiment 1, the main effect of retention interval did not reach statistical significance.

\section{EXPERIMENTS 3A AND 3B}

Experiment 2 replicated the super-LI effect of Experiment 1 , and the effect was modulated by US intensity.
Specifically, high, but not low, US intensity produced super-LI after a 21-day delay. Such a super-LI effect is not readily explained by traditional association deficit theories (see Lubow, 1989, for a review), which make no predictions in regard to the effects of a delay between conditioning and testing. However, retrieval failure interference hypotheses (Bouton, 1993; Miller et al., 1986), at least in principle, may account for such a finding.

One relevant factor might be the role of the context as a retrieval cue. As Bouton (1993) has suggested, in a typical LI experiment, at the time of testing, the context provides a retrieval cue for two different associations: the CS-no-consequence association formed during the pre- 
exposure phase and the CS-US association formed during the conditioning phase. Any manipulation of context during the delay could change the ability of the context to retrieve either one or both of these associations. Indeed, as was noted by Killcross, Kiernan, Dwyer, and Westbrook (1998b), most of the CTA experiments that have examined LI as a function of conditioning-test delays (e.g., Aguado et al., 1994, Experiment 1; Bakner et al., 1991, Experiments 1 and 2) and that found that long delays attenuated LI have used procedures in which preexposure, conditioning, delay, and test phases were all conducted in the home cage. The one study that failed to find LI attenuation with delay (Álvarez \& López, 1995, Experiment 2) also used the home cage for all the procedural phases, but the home cages were placed in a different room from that of the vivarium, and the delay period was spent in the vivarium. In Experiments 1 and 2 of the present study, the super-LI effect was obtained when the delay context was different from that of preexposure, acquisition, and test. Although the findings across experiments are not completely consistent, it would appear that the direction of the effects of delay-namely, attenuation or facilitation of LI-depends, at least in part, on whether the delay period is spent in contexts that are the same as or different from those of the preexposure, acquisition, and test phases. Specifically, when the context is the same during the entire course of the experiment, including a long delay period, the $\mathrm{CS}-$ and/or the US-context associations may extinguish, thereby decreasing the ability of the test context to retrieve one or both of these associations. If, after a long acquisition-test delay, the test context retrieves weak CS-no-consequence and weak CS-US associations, interference between the two associations should be reduced, as compared with the short-delay groups. Since interference between the flavorno-consequence association and the flavor-US association can only occur in the PE groups, the effects of the place-of-delay manipulation should be reflected in the magnitude of LI, but only as a result of differences in test flavor consumption among the PE groups.

On the other hand, when the preexposure, conditioning, and test phases are conducted in a different context than that of the delay phase, then, in the test, the two retrieved associations should be both strong and clearly differentiated from each other. In the test stage, the context, not having undergone an extinction procedure, will have maintained its retrieval capacities, and LI should not be affected by the duration of the delay interval.

To test these hypotheses, we conducted two experiments that used the context conditions for inducing either attenuated LI or super-LI effects. Each experiment was composed of a $2 \times 2$ factorial design, with one variable being preexposure (PE vs. NPE) and the second delay interval between conditioning and test phases ( 1 vs. 21 days). In Experiment $3 \mathrm{~A}$, designed to produce a super-LI effect, the contexts for preexposure, conditioning, and test were the same but were different from that of the delay period, which was conducted in the home cage. These were the same conditions as those in Experiments 1 and 2. In Experiment 3B, designed to produce attenuated LI, all the phases of the experiment, including the delay interval, were conducted in the home cages.

\section{Method}

Subjects. The subjects were 64 naive adult male Wistar rats obtained from IFFA/CREDO Animals for Laboratory Service (32 in Experiment $3 \mathrm{~A}$ and 32 in Experiment $3 \mathrm{~B}$ ). Their weights ranged from 353 to $516 \mathrm{~g}$. All the animals were individually housed in 40 $\times 20 \times 24 \mathrm{~cm}$ Plexiglas cages on a 12:12-h light:dark cycle. All the testing occurred during the $12-\mathrm{h}$ light period. Standard rat food was continuously available.

Apparatus and Procedure. In Experiment 3A, preexposure, conditioning, and testing phases took place in a different room and in different cages from the home cages-the same experimental cages employed in Experiments 1 and 2. The delay period was spent in the home cage. In Experiment 3B, all the experimental manipulations were conducted in the home cages. For both Experiments $3 \mathrm{~A}$ and $3 \mathrm{~B}$, the procedure was the same as that described for Experiments 1 and 2, except that three instead of five extinction trials were administered. The US was a $0.5 \%$ of body weight, $0.4-\mathrm{M} \mathrm{LiCl}$ i.p. injection.

\section{Results and Discussion}

Experiment 3A. On the conditioning day, the mean amount of saccharin consumed by the four groups ranged from 8.49 to $15.46 \mathrm{ml}$. There were no significant differences between groups $[F(3,28)<1]$. As in the previous experiments, subsequent analyses were conducted on the amount consumed on extinction trials.

Figure 3 displays the mean amount of saccharin solution consumed as a function of preexposure and delay for each of the three extinction trials (panel A) and also averaged over the three trials (panel B). A $3 \times 2 \times 2$ ANCOVA, with trials, preexposure, and delay as main variables and amount of flavored solution consumed during the conditioning phase as a covariate, was applied to the data. There was a main effect of trials $[F(2,56)=129.42, p<$ $.001]$ and significant preexposure $\times$ trials and delay $\times$ trials interactions $[F(2,56)=8.73, p<.01$, and $F(2,56)=$ $3.60, p<.05$, respectively]. The interactions reflect the different rates of extinction as a function of preexposure and delay levels. The preexposure $\times$ delay $\times$ trial interaction was nonsignificant $[F(2,56)=1.16, p>.32]$. The main effects of preexposure and delay were significant $[F \mathrm{~s}(1,27)>33.10, p \mathrm{~s}<.001]$, revealing both LI and a reduction in CTA when it was assessed 21 days, as opposed to 1 day, after conditioning. Importantly, the preexposure $X$ delay interaction was significant $[F(1,27)=5.09, p<$ $.05]$, indicating, as in Experiments 1 and 2, a super-LI effect. The interaction is displayed in Figure 3 (panel B), where it can be seen, once again, that the LI effect was larger after a 21-day delay than after a 1-day delay.

Experiment 3B. On the conditioning day, the mean amount of saccharin consumed by the four groups ranged from 9.32 to $16.76 \mathrm{ml}$. There were no significant differences between groups $[F(3,28)<1]$. 

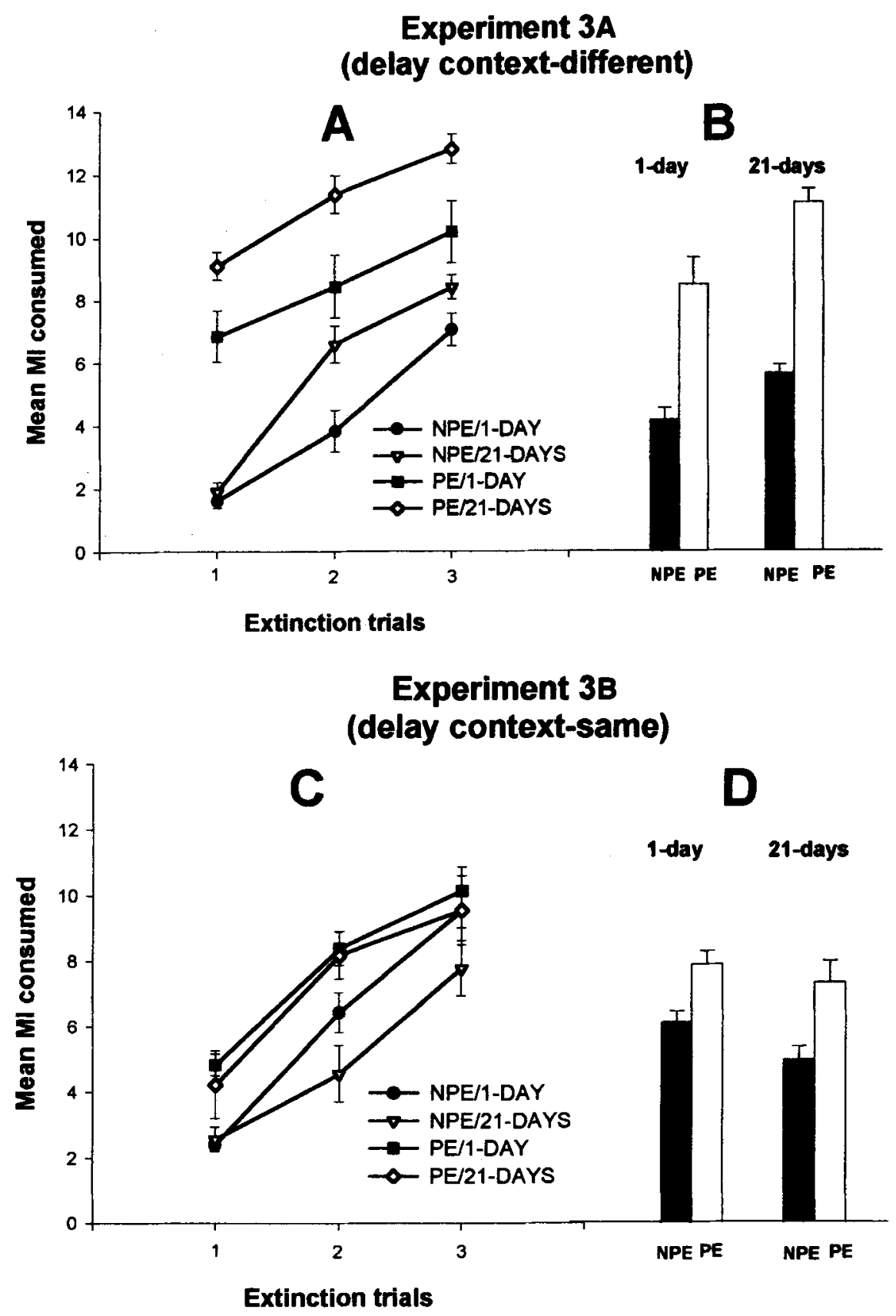

Figure 3. Mean amount of saccharin solution consumed by flavor-preexposed (PE) and flavornonpreexposed (NPE) groups as a function of delay between conditioning and test stages ( 1 and 21 days) for Experiments $3 A$ (upper panels) and $3 B$ (lower panels). The left-hand panels ( $A$ and $C)$ present the data across the three extinction trials. The right-hand panels ( $B$ and $D)$ present the data collapsed across trials.

The bottom portion of Figure 3 displays the mean amount of saccharin solution consumed as a function of preexposure and delay for each of the three extinction trials (panel $A$ ) and also averaged over the three trials (panel B). As in Experiment 3A, a $3 \times 2 \times 2$ ANCOVA was conducted on the mean amount of saccharin solution consumed as a function of trials, preexposure, and delay, with amount consumed in the conditioning phase as a covariate. The main effect of trials was significant $[F(2,56)=84.71, p<.001]$; none of the interactions with trials was significant ( $p s>.16)$. The main effect of preexposure was significant $[F(1,27)=17.51, p<.001]$, revealing the usual LI. Delay was not significant $[F(1,27)=$ $3.31, p>.08$ ]. As can be seen in Figure 3 (panel D), there 
is no evidence for a preexposure $x$ delay interaction. Indeed, the preexposure $\times$ delay interaction was not significant $(F<1)$, indicating that LI was not affected by the duration of the delay interval.

In summary, in Experiment $3 \mathrm{~A}$, when the delay interval was spent in the home cage and the preexposure, conditioning, and test phases were conducted outside the home cage, super-LI again was obtained. In Experiment 3B, when all the experimental stages were conducted in the same place-namely, in the home cages-there was no super-LI. Together, Experiments $3 \mathrm{~A}$ and $3 \mathrm{~B}$ suggest that the delay context modulates the super-LI effect. However, the fact that LI in Experiment 3B was obtained in both the 1- and the 21-day-delay groups and that there was no reduction of LI with delay indicates that the attenuation of LI reported in other studies using the same conditions (Aguado et al., 1994; Bakner et al., 1991; De la Casa \& Lubow, 1995) requires further investigation, in order to identify those conditions that lead to delayinduced attenuation of LI.

\section{GENERAL DISCUSSION}

Unlike in other studies that have shown that LI is attenuated with long, as compared with short, acquisition-test delays (e.g., Aguado et al., 1994; Bakner et al., 1991; De la Casa \& Lubow, 1995), we produced a reliable superLI effect (Experiments 1, 2, and 3A). In all cases, the increase in LI was derived from the fact that the $P E / 21$ - day-delay groups exhibited less CTA (drank more) than did the PE/1-day-delay groups.

As can be seen in Figure 4, which combines the data from Experiments 1 and 2 (collapsed across trials), this finding was, in part, a function of US intensity. With an increase in US intensity, delay, at best, moderately increased the effects of conditioning (compare the NPE/1day-delay group with the NPE/21-day-delay group). However, the retention interval effect, contrary to other reports (e.g., Batsell \& Best, 1992; Batsell \& George, 1996), did not reach standard levels of significance. Importantly, as can be seen in Figure 4, there was an opposite effect in the PE groups. Increasing the intensity of the US increased the effects of stimulus preexposure, thus producing more LI with long than with short delays between the acquisition and the test phases. In brief, Figure 4 suggests, and the three-way interaction in Experiment 2 confirms, that the effects of delay are modulated by US intensity, so that LI (PE minus NPE) in the long-delay group is greater when conditioning occurs with a high-intensity US than with a low one. Nevertheless, since there are two CTA studies that used even higher intensities than our highest and found attenuated LI with delay (Aguado et al., 1994; Bakner et al., 1991), one cannot attribute the super-LI effect to US intensity by itself.

Experiments $3 \mathrm{~A}$ and $3 \mathrm{~B}$ suggest that, in addition to US intensity, super-LI is a function of the relation between the context of preexposure, acquisition, and test and the context in which the delay period is spent. Thus, those

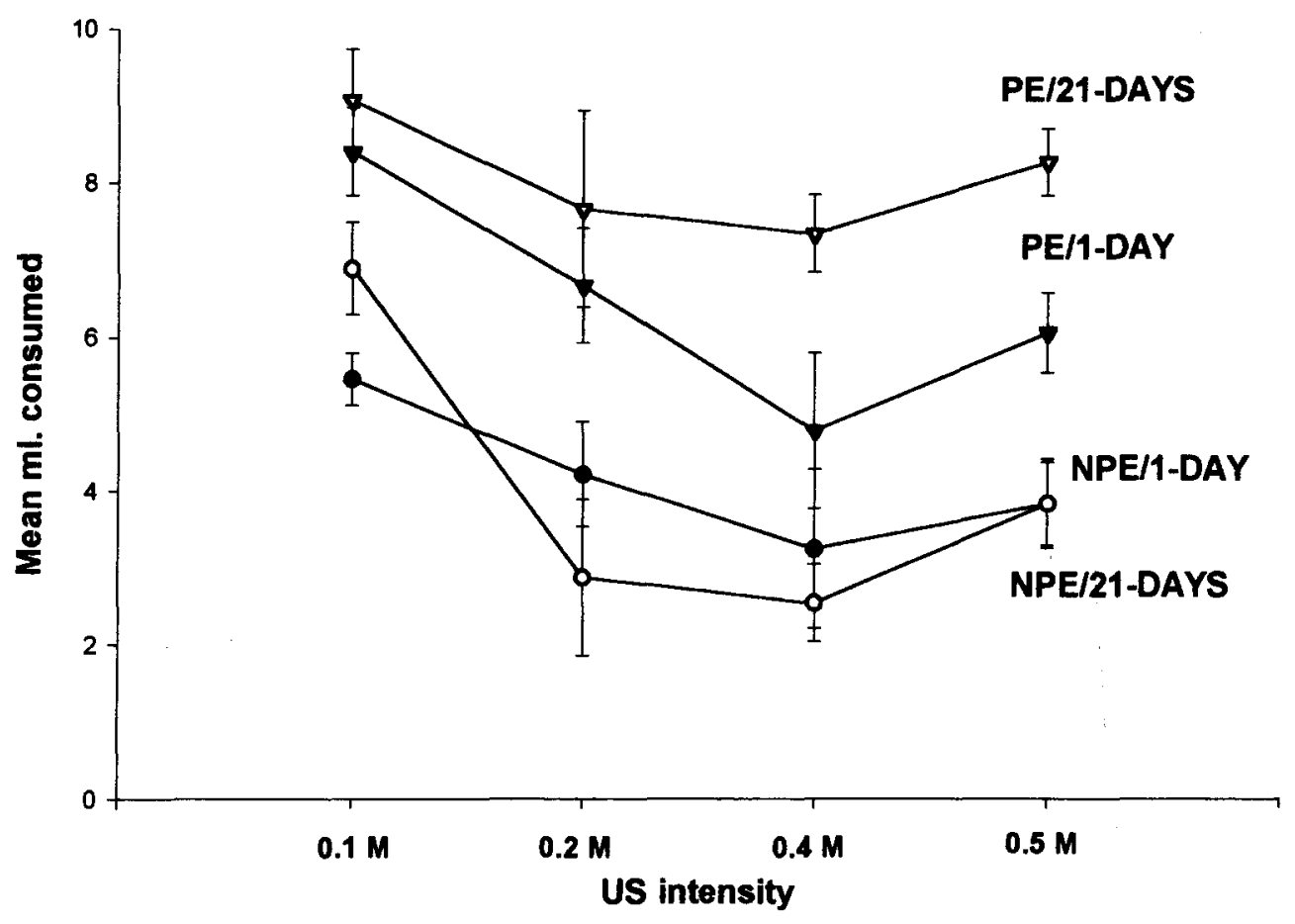

Figure 4. Mean amount of saccharin solution consumed (averaged over extinction trials) as a function of unconditioned stimulus (US) intensity ( $\mathrm{LiCl}$ concentration) by preexposed (PE) and nonpreexposed (NPE) groups, tested 1 and 21 days after conditioning. The data are from Experiments 1 and 2. 
CTA experiments that have reported an attenuation of LI with delay (Aguado et al., 1994; Bakner et al., 1991, Experiments 1 and 2; see also Kraemer \& Ossenkopp, 1986; Kraemer \& Roberts, 1984; Kraemer \& Spear, 1992) have, with one exception (De la Casa \& Lubow, 1995), conducted all stages of the experiment, including the delay, in the same apparatus-namely, the home cage. In the present set of studies, we obtained super-LI when the preexposure, acquisition, and test phases were conducted in a different context from that of the delay phase (Experiments 1,2 , and $3 \mathrm{~A}$ ). We failed to obtain super-LI when the contexts in all the phases were the same as the delay context (Experiment 3B). (Such a conclusion, deriving from a comparison of Experiments $3 \mathrm{~A}$ and $3 \mathrm{~B}$ must, however, be accepted with caution, since there is a confounding of context type and context change across the two experiments.) Interestingly, in the one study that reported a loss of LI with delay, even when the preexposureacquisition-test context was different from the delay context, the amount of flavor preexposure was considerably less than that in the present experiments. De la Casa and Lubow (1995) preexposed saccharin for 2 days, $5 \mathrm{~min}$ each, as compared with 4 days, 5 min each, in the present experiments.

From the above, it would appear that the amount of flavor preexposure, the intensity of the US during the conditioning phase, and the relation of the delay context to the contexts of the other phases (same or different) all modulate the effects of conditioning-test delays. This pattern of findings has implications for association deficit and retrieval interference theories of LI.

Those studies that report LI attenuation with long conditioning-test delays (Aguado et al., 1994; Bakner et al., 1991) do provide evidence that, despite LI with short delays, the CS-US association was acquired in the conditioning phase. As such, those data preclude a strict association deficit interpretation of $\mathrm{LI}$, at least as applied to three-stage paradigms. However, if there is a recovery of the CS-US association in the test phase, despite stimulus preexposure, as these data indicate, how are we to interpret the increase in LI as a function of delay in the present experiments?

Our proposal begins with some standard assumptions of retrieval interference theories. During the preexposure phase, the animal learns a CS-no-consequence association (A) and, in the subsequent conditioning phase, a CS-US association (B). Importantly, the A and B associations share the same CS, and they were acquired in the same context. Let us assume that the two associations are developed independently of each other and that, at the time of test, when the CS flavor is presented, the two associations compete for expression-to drink or not to drink. In this competition, the amount of flavored water that is ingested must represent a compromise solution between $A$ and $B$. That is, it is not a matter of whether $A$ or $\mathrm{B}$ is retrieved, for in that case the data would be dichotomous - that is, the animals either consume an amount of liquid (high) indicating no conditioning (no B associ- ation) or they consume an amount (low) indicating no preexposure effect (no A association). In fact, the amounts ingested do not represent an all-or-none function, thereby indicating that the response reflects a summation of the A and the B associations. If such is the case, there are multiple sources for the compromise response. The test phase response measure may be the outcome of a change in the strength of the A association, a change in the strength of $\mathrm{B}$, or some combination of the two.

Since past studies have reported LI attenuation with delay, it has naturally been assumed that; in the competition between CS-no-consequence and CS-US, it is the first association that, with an increase in delay time, either is weakened or becomes less retrievable (e.g., Killcross et al., 1998b). In keeping with this set of assumptions, an increase in LI with delay, as is reported in the present experiments, would be due to enhanced retrieval of the preexposure episode. The position that the explanatory burden for both attenuated and super-LI effects should be assigned mainly to the CS-no-consequence event is supported by several additional arguments: the primacy principle, the sensitivity of CS-no-consequence association to context manipulations, and the present data.

The primacy principle asserts that first training is disproportionately strong, as compared with subsequent training (for empirical examples, see Bouton \& King, 1986; Reed, Croft, \& Yeomans, 1996; Scavio, Ross, \& McLeod, 1983). The significance of primacy also has been noted by Konorski in regard to the transfer of excitatory responses to conditioned inhibitory responses and vice versa, as well as a more general principle (Konorski \& Szwejkowska, 1952; see also Bouton, 1993; Lubow, 1989, p. 89; Wickens, Tuber, \& Wickens, 1983). However, of particular interest is the finding that when first learning interferes with performance of second learning, a delay between phases increases the proactive interference effect (Bouton, 1993; Spear, 1978).

A comparison of Experiments $3 \mathrm{~A}$ and $3 \mathrm{~B}$, as well as of the present set of studies with those that obtained attenuated LI with delay, indicates that context plays a critical role in the delay-induced modulation of LI. As has been extensively documented by Bouton (Bouton, 1993; Bouton, Nelson, \& Rosas, 1999), CS-nothing effects are more sensitive to a change of context from one phase to another than are comparable tests of the effects of context change on conditioning. This is true whether the CS-nothing trials are presented in the first phase of the experiment, as in LI (e.g., Rosas \& Bouton, 1997), or in the second phase, as in extinction (e.g., Rosas \& Bouton, 1998).

Together, the fact that the LI delay studies use a CSnothing condition and the fact that CS-nothing is in the position for generating a primacy effect suggest that the main locus of delay effects in three-stage procedures relates to the A, and not to the B, association. Given that delay effects are a result of the modulation of the A association, an association that is exclusive to the PE group, what are the roles of US intensity and context manipula- 
tions in generating the delay effects. To begin with, we accept that the test context, when it is the same as the preexposure and conditioning contexts, indeed serves as a cue for retrieval of the competing associations, A and B. However, if the delay period is spent in the same context as the other phases, as the amount of time in the delay context increases, the ability of that context in the test phase to elicit/retrieve the A and B associations is reduced. We propose that such an impairment results from an extinction of the $\mathrm{A}$-context and the $\mathrm{B}$-context associations, with a disproportionate weakening of the A-context, as compared with the B-context, associations. Thus, the context extinction hypothesis accounts for the fact that, in Aguado et al. (1994, Experiment 1) and Bakner et al. (1991, Experiments 1 and 2), there was a delay-induced attenuation of LI, and in our Experiment 3B, there was a failure to obtain a super-LI effect, whereas in Experiments 1, 2, and 3A, there was a super-LI effect (see also Álvarez \& López, 1995, Experiment 2).

The fact that the delay-induced super-LI effect also was dependent on using an intense US suggests that the proposed context extinction mechanism is affected by the discriminability between the preexposure and the acquisition phases. When preexposure, acquisition, and test are conducted in the same context, any manipulation within the preexposure and acquisition contexts that serves to differentiate those phases from each other and from the test phase should have predictable effects on LI. Thus, with a higher US intensity, the conditioning phase is made more discriminable from the preexposure phase and, also, more different from the test phase. As a consequence, the test phase context provides a better cue for retrieving the $\mathrm{A}$ association than for retrieving the $\mathrm{B}$ association. As before, the effectiveness of the retrieval cue would be compromised by a context extinction manipulation.

Finally, although we have used retrieval concepts to explain our data, it should be noted that neither these data, nor those from studies that show an attenuation of LI with delay, provide evidence against association deficit explanations of LI. In the three-stage procedure typically used to study LI with CTA or conditioned suppression, test performance reflects a summation of retrieved associative strengths of $A$ and $B$. This summated value provides no information as to the associative strength of $\mathrm{B}$ at the time of acquisition in the second stage. Nevertheless, and irrespective of its contribution to retrieval deficit and association deficit explanations of LI, the super-LI effect constitutes a new phenomenon, which demands both further empirical scrutiny and theoretical attention.

\section{REFERENCES}

Ackil, J. K., Carman, H. M., Bakner, L., \& Riccio, D. C. (1992). Reinstatement of latent inhibition following a reminder treatment in a conditioned taste aversion paradigm. Behavioral \& Neural Biology, 58, 232-235.

Aguado, L., Symonds, M., \& Hall, G. (1994). Interval between preexposure and test determines the magnitude of latent inhibition: $\mathrm{Im}$ - plications for an interference account. Animal Learning \& Behavior, 22, 188-194.

Álvarez, R., \& LóPEZ, M. (1995). Effects of elements or compound preexposure on conditioned taste aversion as a function of retention interval. Animal Learning \& Behavior, 23, 391-399.

Bakner, L., Strohen, K., Nordeen, M., \& Riccio, D. C. (1991). Postconditioning recovery from the latent inhibition effect in conditioned taste aversion. Physiology \& Behavior, 50, 1269-1272.

BATSELL, W. R., \& BEST, M. R. (1992). Variations in the retention of taste aversions: Evidence for retrieval competition. Animal Learning \& Behavior, 20, 146-159.

BATSELl, W. R., \& GeORGE, J. W. (1996). Unconditioned stimulus intensity and retention interval effects. Physiology \& Behavior, 60 , 1463-1467.

Bouton, M. E. (1993). Context, time, and memory retrieval in the interference paradigms of Pavlovian learning. Psychological Bulletin, 114, 80-99.

Bouton, M. E., \& KING, D. A. (1986). Effect of context on performance to conditioned stimuli with mixed histories of reinforcement and nonreinforcement. Journal of Experimental Psychology: Animal Behavior Processes, 12, 4-15.

Bouton, M. E., Nelson, J. B., \& Rosas, J. M. (1999). Stimulus generalization, context change, and forgetting. Psychological Bulletin, $125,171-186$

De LA CASA, L. G., \& LuBOw, R. E. (1995). Latent inhibition in conditioned taste aversion: The roles of stimulus frequency and duration, and amount of fluid ingested during preexposure. Neurobiology of Learning \& Memory, 64, 125-132.

HALL, G. (1991). Perceptual and associative learning. Oxford: Oxford University Press, Clarendon Press.

Killcross, A. S., Kiernan, M. J., Dwyer, D., \& Westbrook, R. F. (1998a). Effects of retention interval on latent inhibition and perceptual learning. Quarterly Journal of Experimental Psychology, 51 B, 59-74.

Killcross, A. S., Kiernan, M. J., Dwyer, D., \& Westbrook, R. F. (1998b). Loss of latent inhibition of contextual conditioning following non-reinforced context exposure in rats. Quarterly Journal of Experimental Psychology, 51 B, 75-90.

KONORSKI, J., \& SZWEJKOWSKA, G. (1952). Chronic extinction and restoration of conditioned reflexes: IV. The dependence of the course of extinction and restoration of conditioned reflexes on the "history" of the conditioned stimulus (the principle of the primacy of the training). Acta Biologiae Experimentalis, 16, 95-113.

Kraemer, P. J., \& OssenkopP, K.-P. (1986). The effects of flavor preexposure and test interval on conditioned taste aversions in rats. $B u l$ letin of the Psychonomic Society, 24, 219-221.

Kraemer, P. J., Randall, C. K., \& Carbary, T. J. (1991). Release from latent inhibition with delayed testing. Animal Learning \& Behavior, 19, 139-145.

Kraemer, P. J., \& Roberts, W. A. (1984). The influence of flavor preexposure and test interval on conditioned taste aversion in the rat. Learning \& Motivation, 15, 259-278.

Kraemer, P. J., \& Spear, N. E. (1992). The effect of nonreinforced stimulus exposure on the strength of a conditioned taste aversion as a function of retention interval: Do latent inhibition and extinction involve a shared process? Animal Learning \& Behavior, 20, 1-7.

LuBOw, R. E. (1989). Latent inhibition and conditioned attention theory. New York: Cambridge University Press.

LuBOW, R. E., WEINER, I., \& SCHNUR, P. (1981). Conditioned attention theory. In G. H. Bower (Ed.), The psychology of learning and motivation (Vol. 15, pp. 1-49). New York: Academic Press.

Mackintosh, N. J. (1975). A theory of attention: Variations in the associability of stimuli with reinforcement. Psychological Review, 82 , 276-298

Mackintosh, N. J. (1983). Conditioning and associative learning. London: Oxford University Press.

Miller, R. R., Kasprow, W. J., \& Schachtman, T. R. (1986). Retrieval variability: Sources and consequences. American Journal of Psychology, 99, 145-218.

Pearce, J. M., \& Hall, G. (1980). A model for Pavlovian conditioning: 
Variations in the effectiveness of conditioned but not unconditioned stimuli. Psychological Review, 87, 332-352.

ReEd, P., Croft, H., \& Yeomans, M. ( 1996). Rats' memory for serially presented novel flavours: Evidence for non-spatial primacy effects. Quarterly Journal of Experimental Psychology, 49B, 174-187.

Rosas, J. M., \& Bouton, M. E. (1997). Additivity of the effects of retention interval and context change on latent inhibition: Toward resolution of the context forgetting paradox. Journal of Experimental Psychology: Animal Behavior Processes, 23, 283-294.

Rosas, J. M.. \& Bouton, M. E. (1998). Context change and retention interval can have additive, rather than interactive, effects after taste aversion extinction. Psvchonomic Bulletin \& Review, 5, 79-83.

Scavio, M. J., Ross, R. T., \& McLeOD, L. M. (1983). Perseveration of associative strength in rabbit nictitating membrane response conditioning. Animal Learning \& Behavior, 11, 91-94.

SPEAR, N. E. (1978). The processing of memories: Forgetting and retention. Hillsdale, $\mathrm{NJ}$ : Erlbaum.

WAGNER, A. R. ( 1981 ). SOP: A model of automatic memory processing in animal behavior. In N. E. Spear \& R. R. Miller (Eds.), Infor- mation processing in animals: Memory mechanisms (pp. 5-47). Hillsdale, NJ: Erlbaum.

Wickens, C., Tuber, D. S., \& Wickens, D. D. (1983). Memory for the conditioned response: The proactive effect of preexposure to potential conditioning stimuli and context change. Journal of Experimental Psychology: General, 112, 41-57.

\section{NOTE}

1. The effect also has been reported for conditioned suppression (Kraemer, Randall, \& Carbary, 1991). However, the results are difficult to interpret, owing to delay-related differences in baseline. See also a study of conditioned freezing to context stimuli (Killcross, Kiernan, Dwyer, \& Westbrook, 1998a).

(Manuscript received February 24, 2000; revision accepted for publication April 26, 2000.) 